\title{
Produtividade de repolho em função de doses de bokashi
}

\section{Mariana Caroline Guimarães Xavier ${ }^{1}$, Carlos Antônio dos Santos ${ }^{1}$, Evandro Silva Pereira Costa $^{1}$, Margarida Goréte Ferreira do Carmo ${ }^{1}$}

${ }^{1}$ Universidade Federal Rural do Rio de Janeiro, Campus Seropédica, Rio de Janeiro, Brasil. E-mail: marianaacaroline@hotmail.com, carlosantoniokds@gmail.com, evsilvacosta@gmail.com, gorete@ufrrj.br

Recebido: 09/01/2018; Aceito: 14/11/2018.

\section{RESUMO}

Devido às poucas informações sobre a resposta da cultura do repolho (Brassica oleracea var. capitata) a compostos orgânicos do tipo bokashi, desenvolveu-se o presente trabalho, que teve como objetivo avaliar o efeito de diferentes doses de bokashi $\left(0,0 ; 5,0 ; 7,5 ; 10,0\right.$ e $\left.12,5 \mathrm{t} \mathrm{ha}^{-1}\right)$ sobre o seu desenvolvimento e produtividade. Realizou-se ensaio de campo nas condições de Seropédica, RJ, no período de maio a agosto de 2017. Utilizou-se o híbrido Sekai I F1. O composto foi aplicado nas covas de plantio (50\%) e em uma adubação de cobertura (50\%), aos 38 dias após o transplantio (DAT). Adotou-se o delineamento de blocos ao acaso, com quatro repetições. Colheram-se as plantas aos 75 DAT, onde foram obtidas as seguintes variáveis: diâmetro longitudinal (DL) e transversal das cabeças (DT), em cm, massa fresca de cabeças (MFC), em kg, e a produtividade estimada $\left(\mathrm{t} \mathrm{ha}^{-1}\right)$. Os dados obtidos foram submetidos à análise de variância e de regressão a 5\% de probabilidade. Observou-se efeito quadrático das doses de bokashi sobre todas as variáveis analisadas. A melhor dose de bokashi foi de 10,0 $\mathrm{t}$ $\mathrm{ha}^{-1}$, resultando nos maiores valores médios para DL $(12,31 \mathrm{~cm})$, DT $(19,09 \mathrm{~cm})$, MFC $(1,96 \mathrm{~kg})$ e máxima produtividade $\left(32,63 \mathrm{t} \mathrm{ha}^{-1}\right)$. Conclui-se que o composto estimulou positivamente o desenvolvimento vegetativo das plantas, elevando a sua produtividade.

Palavras-chave: Adubação orgânica, Brassica oleracea var. capitata, brássicas, composto.

\section{Cabbage yield as a function of bokashi doses}

\begin{abstract}
Due to the lack of information on the response of cabbage (Brassica oleracea var. capitata) to bokashi type organic compounds, the present work was developed to evaluate the effect of different doses of bokashi $(0.0,5.0$, $7.5,10.0$ and $12.5 \mathrm{t} \mathrm{ha}^{-1}$ ) on their development and productivity. A field trial was carried out in the conditions of Seropédica, RJ, from May to August, 2017. The hybrid Sekai I F1 was used. The compost was applied in the planting pits $(50 \%)$ and in a cover fertilization (50\%), at 38 days after transplanting (DAT). A randomized complete block design was used, with four replications. The plants were harvested at 75 DAT where the following variables were obtained: longitudinal (LD) and transverse diameter of head (TD), in cm, fresh head mass (FHM) in $\mathrm{kg}$, and estimated productivity $\left(\mathrm{t} \mathrm{ha}^{-1}\right)$. Data were submitted to analysis of variance and regression at $5 \%$ of probability. A quadratic effect of bokashi doses was observed on all analyzed variables. The best bokashi dose was $10.0 \mathrm{t} \mathrm{ha}^{-1}$, resulting in the highest mean values for LD $(12.31 \mathrm{~cm})$, TD $(19.09 \mathrm{~cm})$, FHM $(1.96 \mathrm{~kg})$ and maximum productivity $\left(32.63 \mathrm{t} \mathrm{ha}^{-1}\right)$. It was concluded that the compound positively stimulated the vegetative development of plants by raising their productivity.
\end{abstract}

Keywords: Organic fertilization, Brassica oleracea var. capitata, brassicas, compost. 


\section{Introdução}

Dentre as hortaliças folhosas mais produzidas no Brasil, merece destaque especial o repolho (Brassica oleracea var. capitata), por conferir ao pequeno e médio produtor grande importância econômica e social (Filgueira, 2008). Devido ao baixo investimento inicial quando comparado a outras hortaliças, e devido ao retorno a curto prazo, o cultivo do repolho é estratégico e importante para o fortalecimento da agricultura familiar (Sediyama et al., 2014).

A adubação orgânica se apresenta como uma alternativa na redução ou substituição do emprego de adubos químicos e, portanto, torna-se importante a validação e adoção de novas tecnologias e estratégias que possam ser utilizadas na adubação orgânica de hortaliças, como o repolho (Lima et al., 2011). Diversas práticas como o uso de estercos curtidos ou compostados, adubação verde, e a aplicação de compostos, por exemplo, vêm sendo adotadas para suprir a crescente demanda por fertilizantes orgânicos (Siqueira e Siqueira, 2013; Sediyama et al., 2014; Lima et al., 2015).

Nesse contexto, diversos autores têm verificado que a fertilização com compostos orgânicos do tipo bokashi tem sido promissora para uma série de culturas. O bokashi tem como função o fornecimento de nutrientes e microrganismos benéficos ao solo, além da recuperação de áreas nutricionalmente desequilibradas e degradadas (Homma, 2003; Siqueira e Siqueira, 2013; Reis Júnior et al., 2017).

A utilização do bokashi como fonte de matéria orgânica pode aumentar de maneira considerável o rendimento produtivo de brássicas e outras hortaliças, estimulando positivamente o desenvolvimento vegetativo das plantas. Os compostos apresentam como vantagem a possibilidade de adequação pelos agricultores de cada região, de acordo com a disponibilidade de matéria-prima e a finalidade, além da exigência nutricional de cada cultivo, possibilitando um rendimento satisfatório e ecologicamente correto (Lima et al., 2015; Ribeiro et al., 2015; Reis Júnior et al., 2017). Resultados positivos já foram observados por Ferreira et al. (2012) e Saiter et al. (2016) ao avaliarem o efeito do bokashi na cultura do brócolis.

Diante do exposto, e devido às poucas informações sobre a resposta da cultura do repolho ao composto, realizou-se o presente trabalho com o objetivo de avaliar o efeito de diferentes doses de bokashi sobre o seu desenvolvimento e produtividade.

\section{Material e Métodos}

O ensaio foi realizado em condições de campo no Departamento de Fitotecnia da Universidade Federal Rural do Rio de Janeiro - UFRRJ, localizada no município de Seropédica - RJ (22 48'S; 43 $41^{\text {'W }} 33$ $\mathrm{m})$, no período de maio a agosto de 2017. O clima da região, segundo a classificação de Köppen (1948), enquadra-se no tipo Aw - Clima tropical, caracterizado por estação chuvosa no verão, de novembro a abril, e nítida estação seca no inverno, de maio a outubro, sendo julho o mês mais seco. Durante a condução do ensaio, foram observadas temperaturas médias de $21,5{ }^{\circ} \mathrm{C}$, umidade relativa do ar média de $75 \%$ e precipitações que somaram $285 \mathrm{~mm}$ (INMET, 2017).

Foram avaliadas cinco doses de composto orgânico do tipo bokashi: 0,$0 ; 5,0 ; 7,5 ; 10,0$ e $12,5 \mathrm{t} \mathrm{ha}^{-1}$, sendo que o bokashi utilizado foi preparado de acordo com o manual técnico do Programa Rio Rural (Siqueira e Siqueira, 2013). A captura dos microrganismos foi realizada em solo de mata, utilizando-se aproximadamente $500 \mathrm{~g}$ de arroz cozido em água desclorada, o qual foi colocado sob saco plástico e coberto com uma tela fina, sendo posteriormente recoberto com serapilheira. Após 15 dias, as partes mofadas de coloração mais claras, como as alaranjadas e azuladas, foram selecionadas, sendo descartadas as mais escuras, de coloração cinza, marrom e preta. Os microrganismos eficazes foram ativados com a distribuição do arroz em garrafas plásticas de 2 litros, adição de $200 \mathrm{~mL}$ de melaço orgânico e completadas com água desclorada. A solução foi armazenada e checada para liberação do gás produzido e, após 15 dias, quando não havia mais produção do mesmo, estava pronta para uso.

O bokashi foi obtido através da homogeneização de farelo de trigo e torta de mamona na proporção 6:4, 2 litros da suspensão de microrganismos previamente ativados, além de água, dependendo da necessidade. Os materiais foram misturados de forma uniforme e armazenados compactados em tonéis de plástico fechados e, após 21 dias de fermentação anaeróbica, o composto ficou pronto para uso, apresentando as seguintes características: $\mathrm{C}=51,9 \%, \mathrm{~N}=4,2 \%$ e $\mathrm{C}: \mathrm{N}=$ 12,3; $\mathrm{Ca}=9,7, \mathrm{Mg}=6,7, \mathrm{~K}=11,9$ e $\mathrm{P}=8,7$ em g kg${ }^{-1}$; $\mathrm{pH}=4,4$; condutividade elétrica $=2,9 \mathrm{dS} \mathrm{m}^{-1}$.

$\mathrm{O}$ ensaio foi realizado em uma gleba que se encontrava em pleno uso, sendo utilizada anteriormente para o cultivo de mandioca (Manihot esculenta). O preparo do solo se deu no dia 23 de maio de 2017, com aração e, em seguida, gradagem para o nivelamento do solo. O solo da área experimental foi classificado como PLANOSSOLO HÁPLICO e continha as seguintes características físico-químicas: $\mathrm{pH}_{\text {(água) }}=4,6 ; \mathrm{P}=36 \mathrm{mg}$ $\mathrm{dm}^{3} ; \mathrm{K}=51 \mathrm{mg} \mathrm{dm}{ }^{3} ; \mathrm{Al}=0,2 \mathrm{cmol}_{\mathrm{c}} \mathrm{dm}^{3} ; \mathrm{Ca}=1,2$ $\mathrm{cmol}_{\mathrm{c}} \mathrm{dm}^{3} ; \mathrm{Mg}=\mathrm{cmol}_{\mathrm{c}} \mathrm{dm}^{3} ; \mathrm{Na}=0,08 \mathrm{cmol}_{\mathrm{c}} \mathrm{dm}^{3} ; \mathrm{V}=$ $35 \%$.

As mudas de repolho foram preparadas no dia 26 de abril de 2017, em bandejas de isopor de 128 células preenchidas com substrato agrícola comercial e 
mantidas em casa de vegetação. Utilizou-se o híbrido Sekai I F1 (Agristar ${ }^{\circledR}$ ), com ciclo médio de 100 dias, tendo como características o cultivo indicado para o ano todo, tolerância à rachadura da cabeça e alta uniformidade de colheita, segundo o fabricante.

A área foi dividida em 20 parcelas experimentais medindo $9,6 \mathrm{~m}^{2}$, sendo que cada uma foi composta por 16 plantas, utilizando-se o espaçamento de 1,00 x 0,60 $\mathrm{m}$, e totalizando uma área de $192 \mathrm{~m}^{2}$ e 320 plantas. Como parcela útil, consideram-se as quatro plantas centrais de cada parcela, totalizando-se 80 plantas úteis. As plantas utilizadas como bordadura em cada parcela foram submetidas às mesmas condições daquelas pertentes à parcela útil.

O transplantio para o campo foi realizado um mês após a semeadura nas bandejas, no dia 26 de maio de 2017, quando todas as mudas apresentavam dois pares de folhas definitivas. A adubação com o bokashi foi parcelada e realizada em duas etapas: na ocasião do transplantio $(50 \%)$, onde as respectivas doses foram aplicadas em superfície e incorporadas com uso de enxada e, em cobertura (50\%), aos 38 dias após o transplantio (DAT), aplicando-se ao redor das plantas na projeção da copa.

Foram realizadas capinas quinzenais a fim de se reduzir o crescimento e minimizar a interferência de plantas invasoras. Ao longo do ciclo da cultura, o suprimento das necessidades hídricas foi realizado por meio de um sistema de irrigação por aspersão e turno de rega de dois dias, aproximadamente, dependendo da distribuição das chuvas.

A colheita das parcelas úteis foi realizada aos 75 DAT, quando as cabeças se apresentavam compactas e bem desenvolvidas. Realizou-se um corte na base da cabeça, retirando-se as folhas externas a ela, a fim de deixá-las com a apresentação comercial.

Foram avaliados três descritores quantitativos: diâmetro longitudinal (DL) e transversal (DT) (cm), com auxílio de uma régua fixa semelhante a um paquímetro; massa fresca das cabeças (MFC) (kg), com auxílio de balança de bancada. Adicionalmente, a partir dos valores obtidos de massa fresca da cabeça, foi estimada a produtividade da cultura $\left(\mathrm{t} \mathrm{ha}^{-1}\right)$.

Os dados obtidos referentes ao diâmetro longitudinal e transversal, massa fresca das cabeças, além da produtividade, foram submetidos à análise de variância (ANOVA) e à análise de regressão quadrática em função das doses de bokashi $(\mathrm{p}<0,05)$. Utilizou-se o software estatístico SISVAR (Ferreira, 2011).

\section{Resultados e Discussão}

Durante a condução do experimento, não foi constatada a incidência de nenhuma doença, anomalia, ou qualquer outra eventualidade que comprometesse o pleno desenvolvimento das plantas. Foram registradas temperaturas médias de $21,5^{\circ} \mathrm{C}$ e umidade relativa do ar média de 75\% (INMET, 2017), condições tidas como ideais para o crescimento e desenvolvimento das cabeças de repolho (Filgueira, 2008).

Entretanto, as condições iniciais de fertilidade do solo não eram propícias ao cultivo do repolho. De acordo com a análise de solo, o pH apresentava-se fortemente ácido, os teores de $\mathrm{Ca}$ e $\mathrm{Mg}$ estavam abaixo de $3 \mathrm{cmol}_{\mathrm{c}} \mathrm{dm}^{-3}$ e a saturação de bases muito abaixo do recomendado, 70\%. Os teores médios de $\mathrm{P}$ apresentavam-se muito altos e os de $\mathrm{K}$, médios (Filgueira, 2008; Guerra et al., 2013).

Caso a realização de calagem fosse atendida, a produção poderia ter sido incrementada de forma muito acentuada, pois a elevação do $\mathrm{pH}$ para 6,5 aumenta a disponibilidade de nutrientes e favorece o desenvolvimento radicular das plantas (Nunes et al., 1994). No entanto, optou-se por sua não realização como forma de visualizar os efeitos benéficos da aplicação do composto mesmo em condições de solo desfavoráveis.

Foram observadas diferenças significativas para todas as variáveis analisadas, sendo: diâmetro longitudinal (DL) e transversal (DT), massa fresca das cabeças (MFC) e produtividade estimada (Tabela 1), sendo o repolho responsivo à aplicação das diferentes doses de bokashi.

A adubação com bokashi favoreceu o desenvolvimento das plantas, com acréscimos positivos para todas as características avaliadas até a dosagem de 10,0 $\mathrm{t} \mathrm{ha}^{-1}$ (Figuras 1 e 2). Com isso, observou-se efeito quadrático das doses de bokashi sobre todas as variáveis analisadas, com ponto máximo próximo a $10 \mathrm{t} \mathrm{ha}^{-1}$. Observou-se, para a dose de 10,0 $\mathrm{t} \mathrm{ha}^{-1}$, incrementos equivalentes a 46,9\% na massa fresca de cabeças, $20,39 \%$ no diâmetro longitudinal e $20,69 \%$ no diâmetro transversal, garantindo assim 47,04\% a mais de produtividade. A dosagem máxima utilizada (12,5 $\left.\mathrm{t} \mathrm{ha}^{-1}\right)$ proporcionou valores inferiores à anterior $\left(10 \mathrm{t} \mathrm{ha}^{-1}\right)$.

De maneira semelhante, para as variáveis MFC e produtividade, a dose de $10,0 \mathrm{t} \mathrm{ha}^{-1}$ proporcionou os melhores resultados, sendo $1,96 \mathrm{~kg}$ e $32,63 \mathrm{t} \mathrm{ha}^{-1}$, respectivamente (Figuras 2A-B). Com isso, pode-se observar que os incrementos nos diâmetros (Figuras 1AB) e em massa fresca de cabeças (Figura 2A) ocasionados pela aplicação do composto foram essenciais para $\mathrm{O}$ incremento na produtividade observada (Figura 2B), especialmente na dose de 10,0 $\mathrm{ha}^{-1}$.

Os resultados obtidos concordam com os de Guerra et al. (2013), que relatam que a adubação orgânica pode substituir totalmente a adubação mineral, devendo esta prática ser preconizada. Sabe-se que os compostos orgânicos tipo bokashi são ricos em $\mathrm{Ca}, \mathrm{Mg}, \mathrm{K}$ e $\mathrm{P}$ e possuem torta de mamona, rica em nitrogênio. 
Tabela 1. Análise de variância para diâmetros longitudinal e transversal $(\mathrm{cm})$, massa fresca $(\mathrm{kg})$ e produtividade média estimada $(\mathrm{t}$ $h^{-1}$ ) de repolho, cv. Sekai I F1, em função da aplicação de diferentes doses de composto orgânico tipo bokashi. Seropédica, UFRRJ.

\begin{tabular}{lccccc}
\hline \multirow{2}{*}{$\begin{array}{l}\text { Fontes de } \\
\text { variação }\end{array}$} & GL & $\begin{array}{c}\text { Diâmetro longitudinal } \\
(\mathrm{cm})\end{array}$ & $\begin{array}{c}\text { Diâmetro transversal } \\
(\mathrm{cm})\end{array}$ & $\begin{array}{c}\text { Massa fresca } \\
(\mathrm{kg})\end{array}$ & $\begin{array}{c}\text { Produtividade } \\
\left(\mathrm{t} \mathrm{ha}^{-1}\right)\end{array}$ \\
\hline Dose & 4 & $3,7725^{*}$ & $9,0792^{*}$ & $0,50847^{*}$ & $141,34^{*}$ \\
Bloco & 3 & $3,0384^{\mathrm{ns}}$ & $9,1832^{\mathrm{ns}}$ & $0,36359^{*}$ & $100,25^{*}$ \\
Resíduo & 12 & 1,1899 & 2,7848 & 0,07893 & 21,945 \\
\hline CV $(\%)$ & & 9,54 & 9,45 & 17,18 & 17,20 \\
\hline
\end{tabular}

*Significativo a 5\% de probabilidade. ns- Não significativo (* Significant at 5\% probability. ns- Not significant).

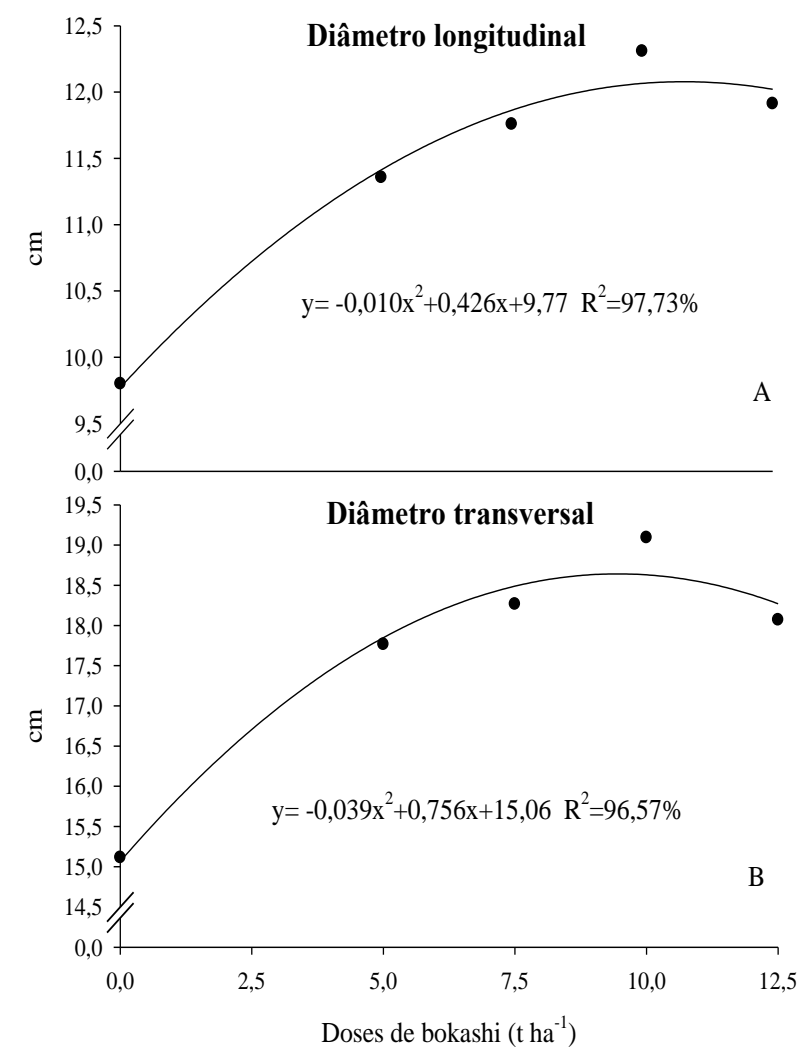

Figura 1. Diâmetro longitudinal (A) e transversal (B) (cm) de cabeças de repolho, cv. Sekai I F1, em função da aplicação de diferentes doses de composto orgânico tipo bokashi. Seropédica, UFRRJ, 2017.

O bokashi utilizado foi resultante da mistura tradicional de farelo de trigo e torta de mamona, e apresentou em sua composição $4,2 \%$ de $\mathrm{N}$, teor superior aos fertilizantes orgânicos tradicionalmente utilizados como o esterco bovino $(2,2 \%)$ e a cama de frango $(2,2 \%)$, o que pode ter colaborado para a obtenção dos resultados positivos anteriormente mostrados (Trani e Trani, 2011; Oliveira et al., 2014). Guerra et al. (2013) recomendam para a cultura do repolho a aplicação parcelada de $60 \mathrm{~kg}$ de $\mathrm{N}$ por ha, portanto, a aplicação de composto, em ambas as doses, atendeu a essa exigência.

Diversos estudos com a utilização de bokashi em doses e formulações variadas demonstraram resultados positivos e aumento do rendimento produtivo de brássicas e outras hortaliças (Ferreira et al., 2012; Souza,

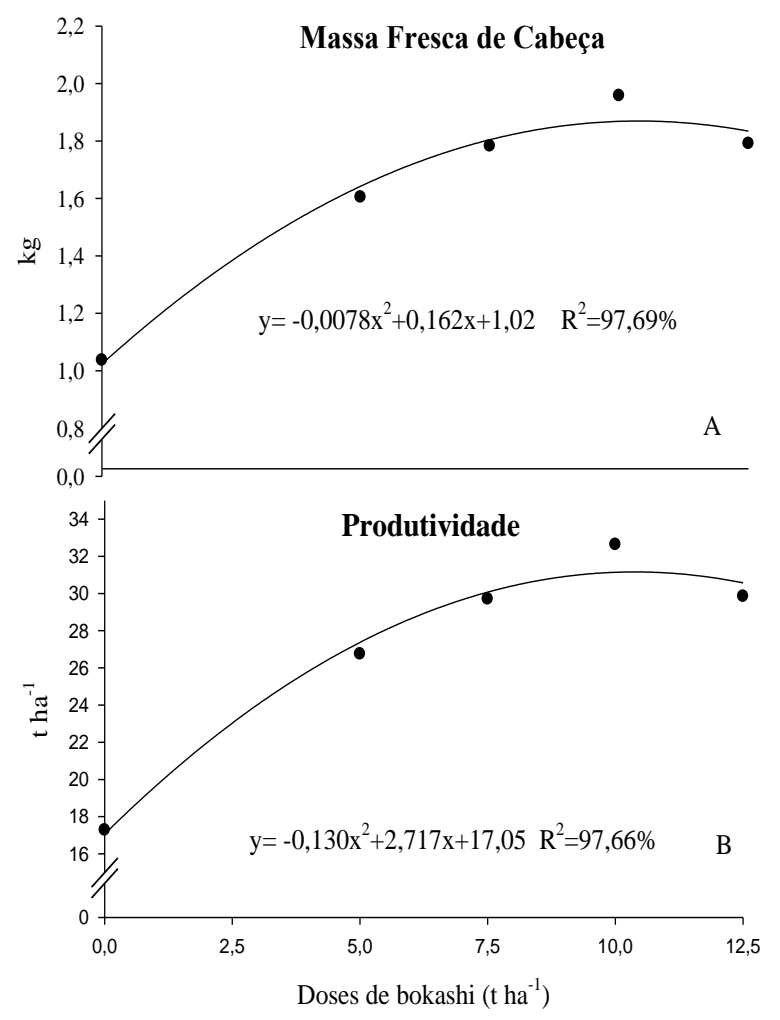

Figura 2. Massa fresca das cabeças (A) (kg) e produtividade estimada (B) $\left(\mathrm{t} \mathrm{ha}^{-1}\right)$ de repolho cv. Sekai I F1 em função da aplicação de diferentes doses de composto orgânico tipo bokashi. Seropédica, UFRRJ, 2017.

2015; Saiter et al., 2016; Silva et al., 2016). Dentre os benefícios da utilização de adubos orgânicos estão o aumento da quantidade e a diversidade da microflora, que favorecem a ciclagem de nutrientes, melhorando assim as condições de fertilidade do solo, além de atuar no controle de pragas e doenças (Siqueira e Siqueira, 2013) como a hérnia das crucíferas, causada por Plasmodiophora brassicae Wor. (Condé et al., 2017). A intensidade de resposta ao bokashi pode ser variável de acordo com fatores como a composição química dos materiais utilizados na fabricação, por exemplo, o teor de $\mathrm{N}$ e $\mathrm{C}$, além da capacidade de resposta de cada cultura.

Resultados semelhantes aos obtidos no presente trabalho foram encontrados por Ferreira et al. (2013), 
que avaliaram as doses $0,2,5,5,0,7,5 \mathrm{e} \mathrm{10,0} \mathrm{tha}^{-1} \mathrm{de}$ bokashi em ensaio com brócolis de verão. Nesse estudo, verificou-se que as doses de bokashi promoveram aumento linear para as características altura de plantas, número de folhas por planta, diâmetro do caule e da cabeça, e massa média de cabeça, até a dosagem máxima utilizada, que correspondeu a $10 \mathrm{t} \mathrm{ha}^{-1}$ (Ferreira et al., 2013).

Condé et al. (2017) observaram que o uso do biofertilizante bokashi $\left(4 \mathrm{t} \mathrm{ha}^{-1}\right)$ resultou em um maior desenvolvimento vegetativo das plantas de repolho, sendo observada a produtividade média de $80,36 \mathrm{t} \mathrm{ha}^{-1}$. As respostas produtivas observadas neste trabalho e as constatadas por Condé et al. (2017) utilizando a dose de $4 \mathrm{t} \mathrm{ha}^{-1}$ foram demasiadamente distintas, devendo-se, provavelmente, às condições iniciais de fertilidade do solo no trabalho dos referidos autores $(\mathrm{pH}$ de 6,5 e saturação de bases igual a 70\%), consideradas mais favoráveis à cultura, além do menor espaçamento adotado.

Nas condições do presente estudo, o uso do bokashi como fertilizante orgânico estimulou positivamente o desenvolvimento vegetativo das plantas de repolho. Com o aumento da dosagem, houve acréscimos na produção de massa fresca e diâmetro de cabeças, elevando a produtividade. Foram observados melhores índices de produtividade com a dose de $10 \mathrm{t} \mathrm{ha}^{-1}$. No entanto, o custo de produção relativamente alto do composto fornece um indicativo da necessidade de uma avaliação mais profunda da rentabilidade da cultura frente ao uso do bokashi, o que não foi contemplado no presente trabalho e que deve ser investigado em novos estudos. Levando-se em consideração R \$ 80,00 como preço médio da saca de $50 \mathrm{~kg}$ de torta de mamona e $\mathrm{R} \$$ 30,00 para a saca de $30 \mathrm{~kg}$ de farelo de trigo, os custos de produção apenas com esses dois ingredientes para cada tonelada do composto seriam de aproximadamente $\mathrm{R} \$ 1.240,00$.

Com isso, torna-se essencial a busca por ingredientes alternativos que visem reduzir os custos de fabricação e que forneçam resultados positivos similares aos dos comumente utilizados, principalmente em substituição à torta de mamona, que, devido ao custo e disponibilidade do material, traz dificuldades para o emprego do composto em grandes áreas.

\section{Conclusões}

A cultura do repolho responde bem à adubação orgânica com bokashi, sendo que a aplicação da dose de 10,0 $\mathrm{t} \mathrm{ha}^{-1}$ resultou na obtenção de plantas superiores para todas as características avaliadas, proporcionando maior produtividade da cultura.

\section{Referências Bibliográficas}

Condé, V. F., Oliveira, D. M., Oliveira, J. E. Z. 2017. Incidência e severidade de hérnia das crucíferas (Plasmodiophora brassicae W.) em repolho (Brassica oleracea L. var. capitata) em solo tratado com biofertilizante tipo bokashi. Ciência e Natura, 39, 07-15.

Ferreira, D. F. 2011. Sisvar: a computer statistical analysis system. Ciência e Agrotecnologia, 35, 1039-1042.

Ferreira, S., Assis, R. P., Souza, R. J., Gomes, L. A. A. 2012. Avaliação da adição de bokashi no cultivo de brócolis Lord Summer. Revista Agrogeoambiental, 4, 1-6.

Ferreira, S., Souza, R. J., Gomes, L. A. A. 2013. Produtividade de brócolis de verão com diferentes doses de bokashi. Revista Agrogeoambiental, 5, 31-38.

Filgueira, F. A. R. 2008. Novo manual de olericultura: agrotecnologia moderna na produção e comercialização de hortaliças. Editora UFV, Viçosa.

Guerra, J. G. M., Leal, M. A. A., Ferreira, M. B. C. 2013. Recomendações de adubos, corretivos e de manejo da matéria orgânica para as principais culturas do Estado do Rio de janeiro: Brócolos, couve, couve-flor e repolho. In: Freire L.R. (Org.). Manual de calagem e adubação do Estado do Rio de Janeiro. Editora Universidade Rural, Seropédica, pp. 107-128.

Homma, S. K. 2003. Nutri-bokashi em respeito à natureza. Fundação Mokiti Okada, São Paulo.

INMET - Instituto Nacional de Meteorologia, 2017. Estações automáticas. http://www.inmet.gov.br (acessado 12 de outubro de 2017).

Lima, P. C., Moura, W. M., Venzon, M., Paula Júnior, T., Fonseca, M. C. M. 2011. Tecnologias para produção orgânica. EPAMIG, Viçosa.

Lima, P. C., Moura, W. M., Almeida, C. H. S., Sediyama, M. A. N., Santos, I. C. S. 2015. Agricultura orgânica e agroecologia: preparo do solo e adubação. Informe Agropecuário, 36, 7-18.

Nunes, M. U. C., Oliveira, J. B., Fazolin, M. 1994. Cultivo de repolho (Brassica oleracea var. capitata) no Acre. Embrapa CPAF, Rio Branco (Circular técnica, 11).

Oliveira, E. A. G., Ribeiro, R. L. D., Leal, M. A. A., Guerra, J. G. M., Araujo, E. S., Espíndola, J. A. A., Rocha, M. S., Bastos, T. C., Saiter, O. 2014. Compostos orgânicos fermentados tipo "bokashi" obtidos com diferentes materiais de origem vegetal e diferentes formas de inoculação visando sua utilização no cultivo de hortaliças. Embrapa Agrobiologia, Seropédica (Boletim de Pesquisa e Desenvolvimento, 98).

Reis Júnior, J. R., Toledo, M. V., Sandri, D. M., Silva, J. C. B. V. 2017. Defensivos alternativos: recomendações práticas para transição agroecológica. Instituto Emater, Curitiba.

Ribeiro, A. P., Santos, A. T. B., Mello, E. R., Barreto, F. G., Nacera, D., Pereira, M. C., Elteto, Y. M., Alves, M. B., Coelho, F. M. G. 2015. Bokashi e EM: "fermentos da vida". UFV, Viçosa. 
Saiter, O., Oliveira, L. A. A., Oliveira, E. A. G., Araujo, D. B. 2016. Efeito do adubo orgânico fermentado bokashi no desempenho agronômico do brócolis americano. Programa Rio Rural, Teresópolis.

Sediyama, M. A. N., Santos, I. C., Lima, P. C. 2014. Cultivo de hortaliças no sistema orgânico. Revista Ceres, 61, 829-837.

Silva, P. N. L., Lanna, N. B. L., Cardoso, A. I. I. 2016. Produção de beterraba em função de doses de torta de mamona em cobertura. Horticultura Brasileira, 34, 416-421.
Siqueira, A. P. P., Siqueira, M. F. B. 2013. Bokashi: adubo orgânico fermentado. Programa Rio Rural, Niterói (Manual Técnico 40).

Souza, J. L. 2015. Agricultura orgânica: tecnologias para produção de alimentos saudáveis. Incaper, Vitória.

Trani, P. E., Trani, A. L. 2011. Fertilizantes: Cálculo de Fórmulas Comerciais. Instituto Agronômico, Campinas (Boletim Técnico, 208). 